\title{
Un bolet méconnu Suillus Boudieri (Quél.) Marchand
}

\author{
ANDRE MARCHAND
}

(Perptgnan; France)

Aux portes de Perpignan, il est une montagnette agréablement boisée de pins oú, pendant tout l'arrière automne et quelquefois jusqu'au printemps, on peut récolter à pleins paniers, un bolet pourtant réputé rare. Tel de nos amis le cueille, en ce lieu-dit Mont-Pins, et le consomme depuis des lustres. Outre son abondance, il offre en effet, une bonne comestibilité, supérieure à celle du Suillus granulatus type.

Les Catalans ne l'ignorent pas! Ils ramassent le champignon avec soin mais le confondent avec les autres bolets des pins sous le nom de "vachette". De fait, il pleure un „lait" plantureux. De plus, le chapeau fortement visqueux et intégralement blanchátre au début, attire vite l'attention du spécialiste sur l'originalité de cette plante curieuse et mal connue.

Le bolet de Mont-Pins correspond, en fait, au Suillus, que sur la Cóte d'Azur et pareillement sous pin d'Alep, Quélet a récolté et nommé Ixocomus Boudieri en 1878. Sans attendre davantage, nous en proposons une description moyenne à cette fin de compléter la diagnose du jurassien, très insuffisante en regard des exigences de la mycologie moderne.

Description. Chapea u de $8-(10) \mathrm{cm}$ de diam., charnu, convexe-hémisphérique puis convexe-plan, \pm bosselé. Ses couleurs présentent une évolution riche en épisodes: d'abord entièrement blanchâtre, puis argilacé terne, il se marbre de beige, de brun-havane et de tonalitès olivâtres pour finir roux-châtain parfois violeté vers les bords. Sur nombre d'exemplaires, la viscosité marquée du revêtement se fige en mèches ou fibres granuleuses et noirâtres, radiales, denses et apprimées au centre oú elles s'anastomosent et prennent du relief, plus éparpillées dans la zone péridiscale sur fond roux plus clair jusqu'ả la marge aiguë, excédante, fortement enroulée chez les jeunes, encore incurvée sur l'adulte, très longtemps blanche, mais jaune-citrin tout à la fin. Cuticule aisément séparable, membraneuse, peu élastique. Stipe plein, ferme, court, en 


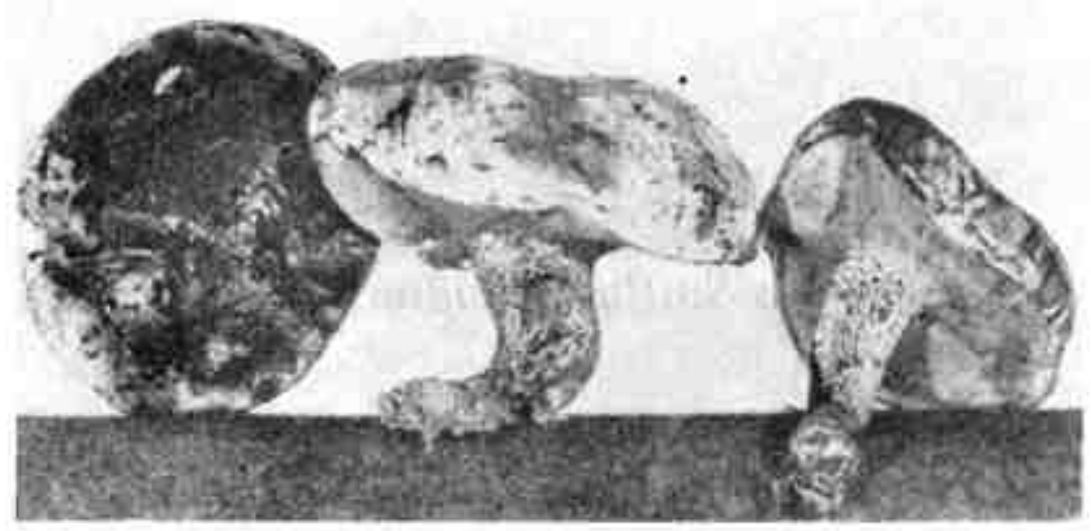

Fig. 1. Suillus Boudieri (Quél.) Marchand. Spécimens cueillis à Mont-Pins, 1.1966. Mise en évidence de la viscositê du chnpealu, de sa couleur blanchàtre au dobbut et de sa msrge excédante. Forme typique du pied tronc-conique, orné de granules.

moyenne $3 \mathrm{~cm}$, parfois très court, par ex. $1,5 \mathrm{~cm}$, presque toujours coudé en bas oú, très souvent, il s'atténue et offre un aspect général tronc-conique, en moyenne $2,2 \mathrm{~cm}$ de diamètre tout en haut pour $1,2 \mathrm{~cm}$ presque en bas. Depuis l'extrême base, un exsudat résinoïde le couvre de granules rouge-grenat passant au brun-rouge noirâtre, ponctiformes à la naissance du stipe, confluant en macules divaricantes vers le milieu tandis qu'ils s'allongent en tirets verticaux dans la partie supérieure où ils se détachent sur fond citrin-vif, alors que le reste du pied demeure

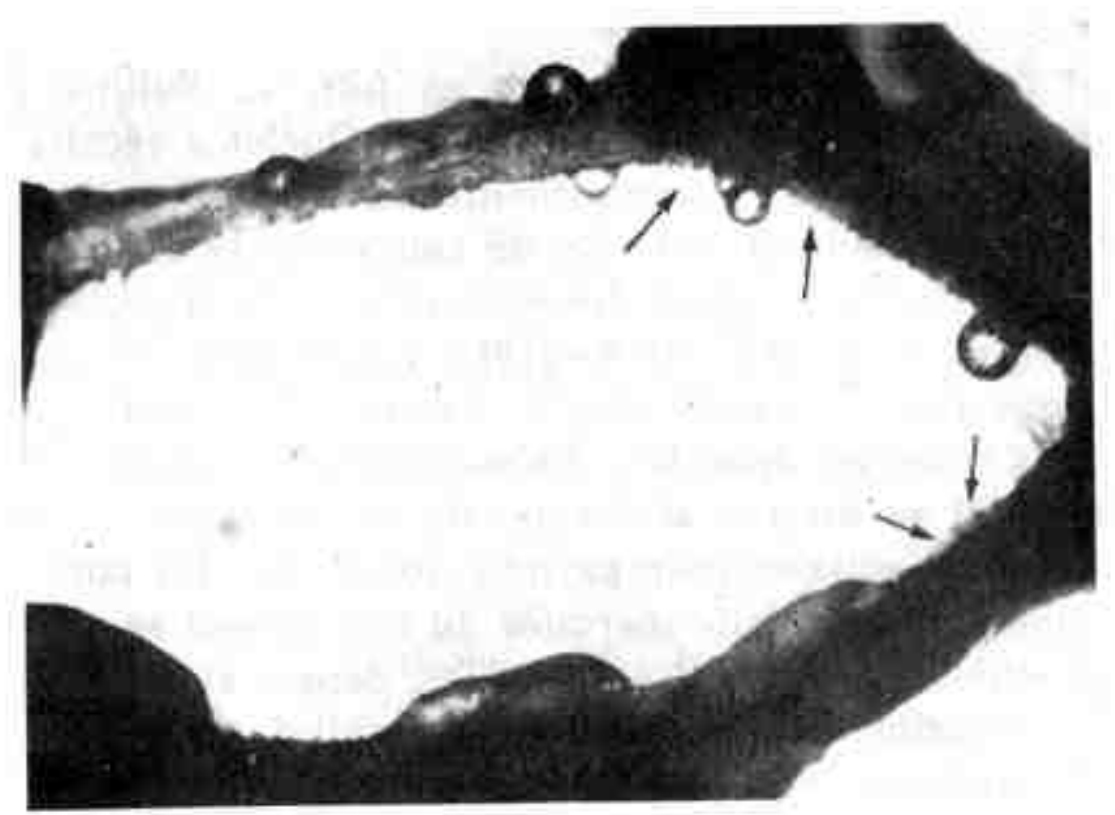

Fig. 2. Suillus Boudieri (Quél.) Marchand. Pore de thymenium. Les fléches montre des cystides en palissades oủ germent les minuscules gouttes d'exsudat en formation. 
blanchâtre, Voile absent. Mycélium abondant, soit blanchåtre, soit subtilement rose-violace, soit encore discrètement marqué de rosâtre-saumoné sur des récoltes effectuées le même jour, à quelques mètres de distunce. $\mathrm{Ch}$ a ir humide, ferme, blanc-nacré jusqu'à la base du stipe, jaune-citrin vers les surfaces, surtout au voisinage immédiat de l'hymenium, non bleuissante. A la longue, le jaune-citrin envahit tout le bas, tancis qu'une zone vineuse apparait sous la cuticule et gagne en direc-

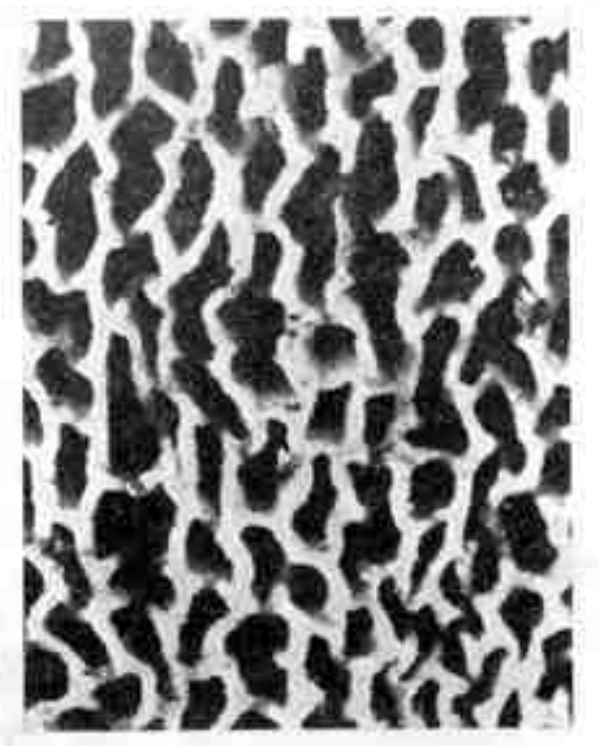

Fot. 3. Sutlus Bondieri (Quél.) Marchand. Pores et lexsudat qui commence à perler à leur orifice.

tion du pied. Odeur forte, fruitée, mélangée à celle de coco, Saveur agréable, fongique, de Marasmius oreades. Tubes nettement décurrents au début, mais adnés sur l'adulte et adnés-sinués à la fin, courts, 8-10 mm, pratiquement non-séparables, jaune-verdătre. Pores simples, petits, moins de $1 \mathrm{~mm}$, anguleux, au début jaunâtre très pâle et terne, quelque peu argilacé-verdātre et subtilement rose-saumoné, enfin jaunátre-olivâtre, non bleuissants à la meurtrissure, englués du même exsudat qui forme les granules résinoides du stipe, rouge-grenat puis brun-sombre à complètement noirâtre surtout vers la marge du chapeau. Sporée chamois, jaunâtre-argilacé, terne. Spores lisses, apicule bien évident, elliptiques-fusiformes, jaune très pâle s. m. $9,25 \times$ $\times 3,75 \mu$ turgescentes, largement guttulées en général et, dans certains cas, seule une mince calotte de cytoplasme subsiste à chaque extrémité de la spore. B a si des tétrasporiques, en massue, $25 \times 6,5 \mu$. C y s t i des et caulocystides très nombreuses a l'extrémité des tubes et sur le stipe oủ elles apparaissent en buissons dans lesquels se forment les granules résinoides. Apparemment identiques $10 \times 50-70 \mu$ allantoïdes ou en massues, à contenu densément granuleux, gris-jaunâtre s. m. H y phes 


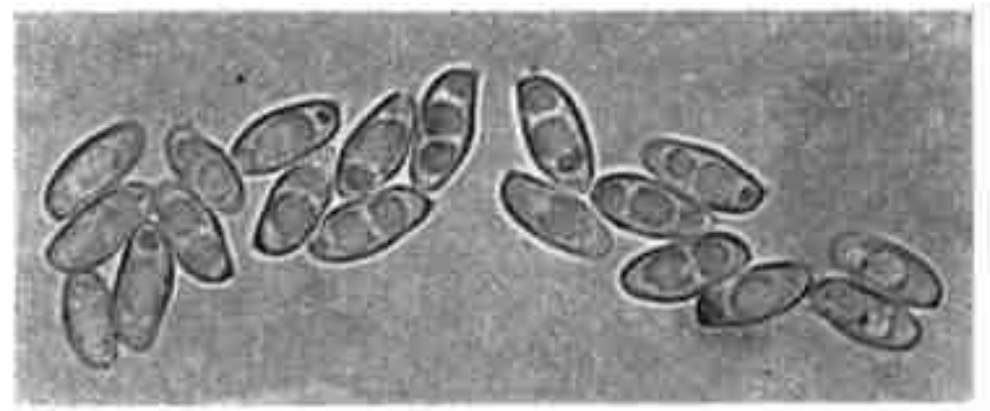

Fot. 4. Sutllus Boudieri (Quél.) Marchand. Spores (2000 *).

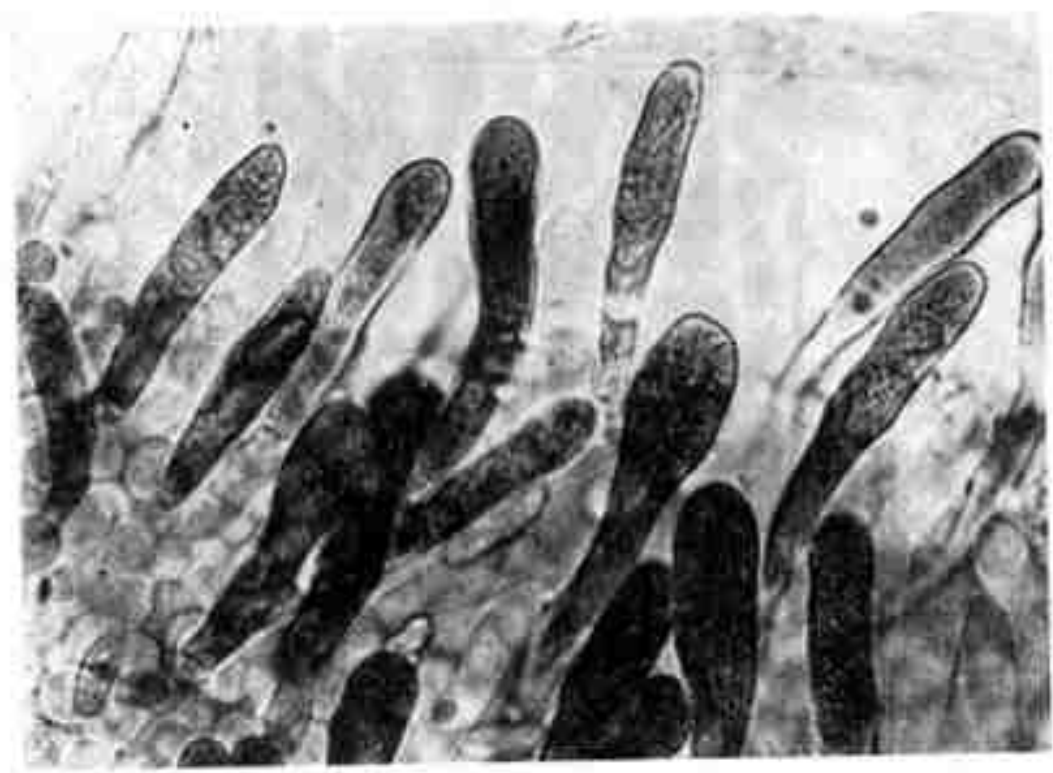

Fot. 5. Suilitus Boudieri (Què) Marchand. Buisson de cystides. La substance résinoide qu'elies contiennent forme les granules de thyménium et du pied.

sans boucles aux cloisons, même à la base des cystides, même à la naissance des basides. Ceux de l'épicutis, 5-6,25 $\mu$ offrent un pigment incrustant très évident qui rend la paroi fortement rugueuse. R éactions chimiques: gaz ammoniac - sur les pores et la chair rouge-cuivré; ammoniaque sur la chair - rose puis bleuattre-livide, enfin lilas; NAOH sur la chair - lilas, sur les tubes - olive puis brun-noiråtre; $\mathrm{FeSO}_{4}$ sur la chair - grisătre-violeté; $\mathrm{H}_{2} \mathrm{SO}_{4}$ sur les tubes rouge puis ocre-jaune; aniline sur la chair - légèrement lilas; formol - néant. Habitat; lieu-dit Mont-Pins à $16 \mathrm{kms}$ NW de Perpignan. Isolé ou grégaire, sous Pinus halepensis d'une jeune plantation. Substrat: schistes albiens. Alt. $80 \mathrm{~m}$. D'octobre à fin janvier, parfois jusqu'au printemps (récoltes notées en février et même un 18 mars. Leg. Joseph Ribalta). 
Pour la clarté de la discussion qui va suivre, nous croyons nécessaire de placer sous les yeux du lecteur la propre diagnose de $Q$ u éle t (1888): Ixocomus Boudieri - „Stipe blanc, lavé de citrin au sommet, pointillé de grains gélatineux, rouge-sanguin. Peridium convexe (OmI), glabre,

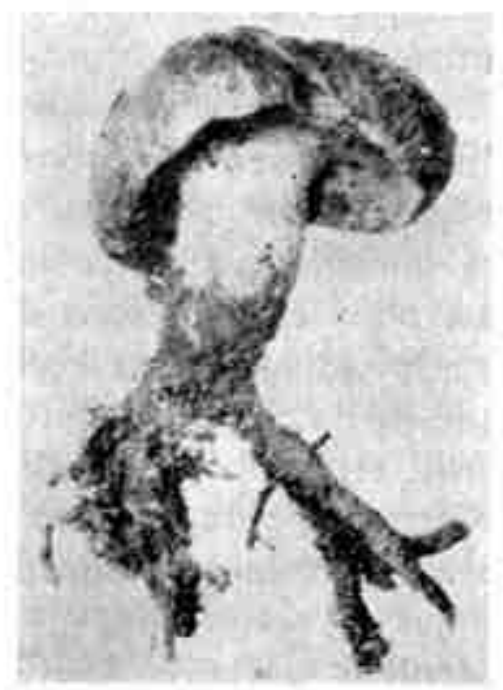

Fot. 6. Boletus Bellinź Inz Spécimen provenant d'israel. Le stipe est terminé par de spectaculaires prolongements radiciformes (R efchert 1940).

blanc, puis violeté ou brunâtre, avec le bord citrin. Chair humide, blanche, puis citrine, douce. Tubes décurrents et pores amples, sinueux, jaune - souci pâle, puis bistre - olivâtre, tachetés comme la stipe de grains résineux et rouges. Spores (OmmOI).

Quél. Sic. bot. 1878, t. 3, f. 3. Bellini, Inz. Sic, t. 6, f. I-5.

Fin automne. Sous les pins d'Alep, aux environs de Menton et de Nice".

\section{DISCUSSION}

A. Identité du bolet de Mont-Pins et d'Ixocomus Boudieri Quél.

L'icône première (Bull. Soc. bot. Fr. 1878, Pl. IIl, fig. 3) offre par une coilncidence remarquable une totale identité d'aspect avec les spécimens qui figurent sur nos propres photographies.

De plus, nous devons à la sollicitude de M. M. Josser and une aquarelle de sa main qui reproduit la pl. 130 des Tabulae analyt. Fung. de Patouillard (1883-89). D'emblée, et sans aucun doute possible, nous reconnaissons notre bolet. Comme Quélet, Patouillard note: „B. Boudieri, environs de Nice sous les pins”. Il avait done la certitude d'avoir retrouvé, et presque au mème endroit, la plante de Quélet.

M. Josserand que nous ne saurions assez remercier pour tant de 
recherches, a pu consulter à la Soc. Linnéenne de Lyon, les Icônes de Brèsadola, et singulièrement la pl. 908. „Mème port, écrit-il, que celui de Patouillard. J'ai tout à fait l'impression que, là encore, on est en présence d'une représentation (assez acceptable) de votre espèce".

Cependant, a elles seules, ces références iconographiques, ne suffiraient pas à établir l'identité du bolet de Mont-Pins et d'Ixocomus Boudieri Quél. Nous demanderons donc a une analyse point par point de la diagnose de Quélet confrontée avec nos propres observations, d'établir que notre plante ne saurait recevoir un autre nom.

Pour respecter l'ordre dans lequel notre auteur attaquait toujours la description des espèces, nous examinerons d'abord comment il rend compte des caractères du stipe, d'autant qu'il s'agit là de la partie peut-être la plus caractéristique de notre bolet. Tout concorde avec nos observations, mais on chercherait en vain des précisions sur la longueur ou l'épaisseur de ce pied. Rien non plus sur son aspect conique, le plus souvent déjeté latéralement, parfois virguliforme, ni sur l'aspect morphologique des granules qui le ponctuent, ni sur le mycélium qui le prolonge, mycelium de couleur variable selon des facteurs de nature. édaphique.

En fait, avec une espèce dont la plasticité des formes, la mutabilité des couleurs proposent une sorte de protée, on regrette, qu'à l'instar de bien d'autres au demeurant, Quélet fige sa diagnose de telle sorte que le processus évolutif ne soit plus sensible. L'insuffisance est lá, plus que dans le laconisme.

Une description dynamique montrerait qu'au début, le développement du chapeau prend du retard sur celui du stipe, A ce moment, la longueur de celui-ci peut égaler le diamètre de celui-lả. Mais l'élan normal de cette croissance du pied perd bientôt de sa vigueur, sauf encore un peu dans le haut, d'oú l'aspect parfois étiré des granules dans cette région, tandis que ceux de la base restent ponctiformes, et ceux de la partie médiane s'étalent en mouchetures qui peuvent s'agréger en plaques maculiformes mais non réticulées, Quant au chapeau, au sujet duquel nous devons revenir, il continue à se développer pour atteindre $10 \mathrm{~cm}$ de diam. mais beaucoup plus souvent $8 \mathrm{~cm}$ quand le pied n'en mesure que 3 . En relation avec la croissance de celui-ci, les tubes tout au début adherent au stipe le long duguel même ils descendent. Mais quand le pied entame sa dernière période de croissance, sa partie supérieure repousse les tubes et pénètre dans l'hyménium. Le champignon acquiert l'aspect de la maturité, et présente alors des tubes adnés puis adnés-sinués.

A l'égard du chapeau, Quélet note des caractères essentiels, donne une dimension, signale la couleur blanche au début et la marge qui devient citrin. Toutefois la même remarque s'impose. La complexe évo- 
lution des couleurs, telle que nous nous efforcons de la restituer, manque, Dans toute sa diagnose, Quélet emploie certes à trois reprises le mot .puis" qui étale quelque peu les métamorphoses dans le temps. Mais on en mesure la carence. Enfin, la forte viscosité de la cuticule n'est pas mise en évidence, ni l'aspect pourtant très typique de la marge excédante,

La chair est effectivement blanche et douce, mais d'un blanc satiné. Longtemps après la coupe, elle devient citrin comme le signale encore Quélet. Pourtant le changement de couleur s'opére graduellement, et une teinte vineuse se manifeste dans le chapeau a partir de la zone sous cuticulaire. Quélet enfin, ne s'urrête ni à l'odeur pourtant forte et assez curieuse avec sa fine pointe de coco, ni à la bonne saveur fongique.

Quand il détaille les caractères des tubes et des pores, il énumère l'essentiel de ce que nous retrouvons dans le bolet de Mont-Pins. Quelques petites divergences intéressent la largeur des pores que, pour notre part, nous jugeons moins amples, leur nuance très terne qui nous a frappé comme la couleur terne du chapeau, enfin la décurrence des tubes qui n'existe qu'au début. Nous répétons, car le point est d'importance, comme on verra plus loin, qu'à taille adulte et jusqu'à la fin, ce bolet montre des tubes adnés, puis adnés-sinués quasiment inséparables du chapeau.

Comme à propos du stipe, Quelet mentionne sur les pores la présence de „grains résineux et rouges". Il n'en dit pas l'origine. Pourtant, l'examen microscopique met en ếvidence, à la base de chaque granule, des touffes de cystides si hautes, si pressées, que le mot „buisson" nous semble relativement plus convenable et plus frappant. A l'intérieur de chaque cystide on distingue une substance obscurément pigmentée et granuleuse dont elle se trouve toute gonflée. La concomitance des cystides et des granules, l'identitè d'aspect s.m. entre le contenu des cystides et les gouttes qui ponctuent le pied et l'hymenium, conduisent à penser que l'exsudat trouve son origine dans les cystides, qui se comportent comme des macrocystides lacticifères. Leur présence en buisson, l'abondance de l'exsudat, qui, hormis le chapeau, macule toute la plante, soulignent le rôle capital qu'elles jouent dans le métabolisme de ce champignon.

Pleuro- et caulocystides travallent chacune comme autant de minuscules alambies qui distillent le latex. De sorte que l'observation macroscopique confirme l'investigation microscopique. Dès qu'on manipule le bolet de Mont-Pins, les doigts sont poissés comme par la résine des branches de Pinus halepensis dont on croit retrouver jusqu'à lodeur. La nature résinoìde de l'exsudat, l'abondance de la nappe mycélienne, donnent à penser que lassociation mycorhizique avec larbre (pin d'Alep ou autre), s'opère de façon très étroite. Enfin nous signalerons 
un autre fait évident: le „vieillissement" de l'exsudat s'effectue de la marge du chapeau en direction de la base du pied.

Au cours de la phase anabolique du champignon, tous les granules présentent une couleur et une consistance qui rappelle le caramel au lait. Au cours de la phase catabolique, et surtout dans la zone antémarginale de l'hyménium, gouttelettes qui ont conflué à l'extrémité de chaque tube et matérialisent chaque pore en le dessinant, forment une résille noire dont la substance évoque le sucre brủlé. Pourtant, à ce moment mème, les granules qui ornent l'extrême base du stipe demeurent rouge grenat, si les autres situés plus haut, sont passés au brun-sombre.

Quélet achève sa diagnose sur une sèche mesure de la spore dont il ne considère que la longueur et qu'il évalue à $10 \mu$. Si, pour notre part, nous donnons des nombres avec des décimales, $9,25 \times 3,75 \mu$ c'est pour la raison qu'ils expriment des moyennes portant sur de très nombreuses mesures que nous pourrions produire hors-texte. Pareillement, nous estimons le diamètre du chapeau à $8 \mathrm{~cm}$, et la longueur du pied à $3 \mathrm{~cm}$, mesures que d'aucuns jugeront trop absolues.

Ce long parallèle entre la diagnose d'Ixocomus Boudieri (Quél.) et notre propre description du bolet de Mont-Pins dégage une certitude bien que nous ayons signalé sans aucune complaisance toutes les lacunes de la première. Indépendamment de notre analyse chacun peut reprendre et comparer les deux textes. La même conclusion s'imposera de soi-méme: le bolet de Mont-Pins est d'Ixocomus Boudieri Quél.

Aux caractères principaux tout à fait identiques s'ajoute le fait que presque à la mẻme latitude (aux environs de Nice), et comme nousmême Quélet a récolté sa plante sous le couvert des Pinus halepensis. Enfin, la data de sa cueillette, en novenbre, offre une ultime concordance. Ixocomus Boudieri Quél., champignon à vocation tardive, pousse tout l'hiver et parfois jusqu'au printemps, malgré le froid, pour peu qu'il pleuve de temps à autre.

Ainsi le nom notre plante sembles assuré par le travail de Quélet. Si on récusait sa diagnose pour ce qui lui manque, le fameux laconisme quélétien conduirait à rejeter toutes les créations du jurassien. Qui s'en aviserait?

Par respect de la tradition et des maitres nous conserverons à notre bolet le nom que son créateur lui a donné.

B. Individualit é d'Ixocomus Boudieri Quél, parmi les esfèces voisines. La confusion extrême qui règne dans la nomenclature des "granulati" provient du fourmillement des noms attribués à des espèces qui se recouvrent, ou à de vagues formes, ou quelquefois même à de simples lusus météoriques. Nous tenons que l'avantage de la mycologie, au 
niveau des possibilités humaines, doit moins s'alourdir dans la prolifération que se clarifier par décantation, fủt-ce au risque de s'appauvrir aux seules espèces parfaitement établies.

Ixocomus Boudieri Quél. en est une, indiscutablement.

Pour lindividualiser parmi celles qui l'avoisinent au plus près, il nous reste à dégager les limites intrinsèques et extrinséques de Boletus leptopus Pers., B. littoralis Bouchet et B. Bellini Inzenga.

B. leptopus Pers. Il prolifère sous le couvert des Pinus pinea de Catalogne. Mais nous en dirons relativement peu de chose ici. Il s'agit d'une espèce mal connue qui mérite aussi une description en forme accompagnée enfin, d'une solide documentation photographique.

En effet, beaucoup de mycologues ont accoutumé de mettre en synonymie B. leptopus Pers. et Ixocomus Boudieri Quél. pour la raison, en particulier, que ce dernier porte un pied court. En réalité Boletus leptopus Pers, correspond à un vrai suille méditerranéen. Ainsi échappet-il au terrains de chasse de la plupart des spécialistes des bolets.

Variété du $B$. circinans de Persoon que le Hollandais créa au début du XIXe siècle après l'avoir récolté, non pas dans les Pays-Bas mais sous nos latitudes, en Italie, B. leptopus Pers. présente des tubes si longuement décurrents quand il est parasité (phénomène très fréquent) qui confère au champignon un aspect turbiné figurant un ..V" majuscule. Le pied excède à grand peine 1 ou $2 \mathrm{~cm}$ de long. et n'offre qu'une granulation discrète à sa surface. Ajoutons, que le chapeau n'est jamais blanc, pas même au début, que la chair réagit â l'ammoniaque en devenant pourpre, comme réagit celle du "granulatus" type de Fries, et que l'hyménium didymoporé s'orne d'un lacis de lignes concolores aux pores qu'elles dessinent et ce qui parfait l'entière originalité de ce bolet. On ne saurait l'assimiler à Ixocomus Boudieri Quél.

B. littoralis Bouchet. Par rapport à Ixocomus Boudieri Quél, autant les caractères de Boletus leptopus Pers. s'affirment singuliers, autant ceux de $B$. littoralis Bouchet (1958) apparaissent voisins, pour ne pas dire identiques. Aussi bien, il nous semble expédient de noter les seules différences, aussi minces soient-elles. Par la taille, le $B$. littoralis évoque un champignon plus grand que l'Ixocomus Boudieri type, puisque le diamètre de son chapeau peut atteindre $15 \mathrm{~cm}$. De même, le stipe se développe davantage pour mesurer $40-50-100 \times 15-25 \mathrm{~mm}$. Dans une de ses lettres, M. Bouchet nous précise encore: „stipe de longueur égale au diamètre du chapeau ou légèrement moindre". Toute chose gardée, la spore accuse en conséquence cette augmentation générale des cotes qui la porte à $9-13 \times 2,5-3,5-4 \mu$. Dernière ,ddifference", mais a retenir, $B$. littoralis Bouchet vient sous le couvert des Pinus maritima. 
La description qu'en donne M. Bouchet comporte quelques lacunes qui concernent la marge du chapeau, est-elle excédante? le mycélium, quelle est son importance? sa couleur?, la disposition des tubes par rapport au pied, sont-ils décurrents, adnés ou libres? Le sont-ils tour à tour? Et M. B o u chet $(1958$, p. 33) ètonne grandement son lecteur quand il écrit, à propos d'un bolet qui exsude tant de latex: „Je n'ai pas vu de cystides sur les préparations examinées (tubes et pores)". Aucune photographie ne vient illustrer son texte, pas mème un dessin au trait.

Cependant nos propres photograpies accompagnant un envoi de matériel frais ont été examinées, pour comparaison, par M. Bouchet. D'emblée celui-ci a reconnu son Boletus littoralis dans nos bolets de Mont-Pins, et il conclut: .les spécimens et votre photo en noir convenaient très bien pour ceux que je récolte ici... Je pense, gråce aussi aux renseignements fournis sur votre lettre, que vas bolets du groupe splorans n'offrent pas de différences fondamentales avec ceux que j'ai recoltés ici" (en Charente Maritime).

D'autre part, notre collègue A. G. P a r rot, a retrouvé ce B. littoralis aux environs de Biarritz et nous a très aimablement communiqué des photographies en noir et blanc ainsi que des diapositives en couleurs fort réussies. Parallèlement à M. Bouchet, nous identifions formellement ce bolet à nos récoltes.

Enfin, M. Romagnesi qui reçut nos bolets de Mont-Pins à plusieurs reprises et dans un état ..présentable", les assimile, Jui aussi, à B. littoralis Bouchet, à condition ,que les peluches du stipes soient bien d'abord rougeâtres". Elles le sont. Grâce à M. Romagnesi qui nous mit en relation avec M. Bouchet et M. Parot notre etude pouvait commencer. Qu'il en soit chaleureusement et très cordialement remercié.

Ainsi tout concourt à rapprocher très étroitement le $B$. littoralis Bouchet d'Ixocomus Boudieri Quél. auquel nous identifions nos bolets de Mont-Pins. Le bolet de M. Bouchet correspond à une variété associée aux Pinus pinaster, plus robuste du fait de thumidité qui favorise la façade atlantique de la France. Mais comme $I$. Boudieri c'est une espèce hivernale qu'on récolte jusqu'aux gelèes (voire entre deux gelées).

Son aire de répartition se révèle fort vaste puisque $M$. Leclair de Bellème nous a confirmé qu'il l'avait cueilli, ,dans les pinèdes sarthoises (sous Pinus maritima), à quelques kilomètres du Mans". Un autre de nos correspondants, M. L. Rallet nous écrit: „Je le croirais volontiers identique à ce qu'on avait nommé antérieurement Boletus Boudieri et dont j'avais vu et peint des exemplaires du Morbihan".

Ainsi répandu bien au Nord de la Loire, nous présumons qu'il existe sur la côte cantabrique et dans les pinèdes lusitaniennes. Nous avons mème des raisons de penser qu'il se rencontre en Amérique. Nous ferons le nécessaire pour le prouver. En tout état de cause, en nous référant 
aux seuls jalons indiscutablement connus nous pouvons avancer que par la vaste répartition de sa variété littoralis, le Boudieri de Quélet est le moins méditerranéen de tous les suillus de la Méditerranée.

B. Bellini Inz. Reste le plus mystérieux des granulati. Souvent on le cite sur la foi de nombreux auteurs qui attribuent son nom, bien entendu, á des espéces différentes quand, par fortune, on peut parler d'espèces! Après son inventeur et Saccardo (1915-16) qui en parle fidèlement, seul ả notre connaissance, Reichert a consacré un travail assez long au B. Bellini d'Inzenga (Fot. 6).

Grâce encore à M. M. J osser and nous avons eu en mains les deux opuscules de Reichert (1940).

Reichert rejette le terme Ixocomus créé en 1888 par Quẻlet. Les suillus se voient divisés en deux groupes. Celui des espèces pourvues d'un anneau reçoit le nom de Boletus employé par Fries en 1821, et celui des espéces sans anneau prend le nom de Rostkovites, créé en 1879 par Karsten.

Toutes les espèces et variétés décrites ici prennent place très près les unes des autres dans la nomenclature. Boletus Bellini Inz. ressemblerait donc vivement á Ixocomus Boudieri Quel. Aussi, pour ne pas changer de méthode nous mettrons surtout en évidence ce qui séparerait les deux espéces, étant entendu que tout le reste concorderait, et qu'il suffit de se reporter à notre description de Boudieri pour en prendre connaissance. Nous laissons à Reichert l'entière paternité de ce qui suit. Le fait qu'il édifie toute sa thèse sur un seul spécimen, tiré d'une station aujourd'hui détruite, suscite le doute et même la suspicion.

$B$. Bellini Inz. offre tout dabord un chapeau convexe et non étalé de 4 à $6 \mathrm{~cm}$ de diam, c'est à dire plus petit que celui d'Ixocomus Boudieri Quél. Reichert (1940) note sa couleur brun noisette léger, plus pâle à la marge qui devient concolore au chapeau, et non pas jaunecitrin comme chez $L$. Boudieri.

La marge apparait plutỏt courte, parfois mème en retrait par rapport aux tubes qu'elle découvre alors. Les pores sont plus petits donc plus nombreux au centimètre carré, et simples. Nous rappelons que nous n'avons pas trouvé de pores composés dans I'Ixocomus de Quèlet, dont la marge, quant à elle, déborde avec netteté du chapeau.

Les tubes descendent le long du stipe „par 2 ou 3 couches d'éléments" au lieu de finir adnés-sinués. Au sujet des cystides, Reichert s'arrête longuement sur un travail statistique qui les fait apparaitre d'une longueur constamment plus étroite que celles de Boudieri. Tel J. Sc h a effer (1952), Reichert attache une grande importance a ce caractère qui, selon lui, distingue tout à fait les deux espèces.

Mais leur disparité s'accuse définitivement quand on compare la base de leur pied. Aussi Reichert s'insurge-t-il contre Gilbert, Maire et 
Singer qui ont négligé dans le $B$. Bellini d'Inzenga ce qu'il décrit ainsi: „The stipe ends in a rootlike base which branches out into finger-like processes, the entire root measuring $6 \mathrm{~cm}$ in length and, at its proximal end, $1-1,5 \mathrm{~cm}$ in width, and the proceses being $2-8 \mathrm{~cm}$ wide". Mais dans sa diagnose du 3.9.1876, Inzenga ne parle que de la base „ove svolgonsi filamenti alquanto solidi, semplici o ramosi di forma radicellare".

De fait, la photographie de Reichert montre un stipe terminé par de spectaculaires prolongements radiciformes, trop ramifiés pour que nous parlions de pseudorhize, trop gros, trop courts pour que nous les assimilions à des rhizomorphes. Si évident qu'apparaisse le mycélium filamenteux, sorte de chevelu radicelliforme, d'Ixocomus Boudieri, il faut convenir qu'll diffèrerait de ce puissant appareil souterrain qui ferait de Boletus Beltinĩ une plante visiblement organisée pour s'accommoder d'une sècheresse sévère.

Son aire de répartition, à ce jour encore circonscrite à la Sicile, où Inzenga l'a trouvé, et à la Palestine où Reichert l'a redécouvert, les sols arénacés dont il se satisfait comme du couvert exclusif des Pinus halepensis, ajoutent trois particularités à toutes les précédentes et feraient de Boletus Bellini Inz, un suille typiquement méditerranéen.

Ceux qui confondent ces deux espèces en une seule, se heurtent à un problème d'appellation. Quel nom retenir? Bellini ou Boudieri? Lequel des deux requiert l'antériorité? Dans un bon article $\mathrm{K}$ on rad (1927) redresse une erreur de date et accorde la priorité à Bellini. Reichert n'en tient pas compte, bien qu'il connaisse cet article puisqu'il le cite dans ses ,references".

En fait, Quélet (1878) crée son Ixocomus Boudieri en 1878. Inz eng a (1879) ne donne l'essor à ses „Funghi Siciliani" qu'en 1879, soit un an plus tard. Toutefois il publie $(1869$, p. 217) le mémoire où il décrit son Boletus Bellini dès 1878, ,but a little later than Quelet's description of B. Boudieri", selon Reichert qui attribue ainsi la priorité a Boudieri.

On comprendra que cette contestation soit sans objet dès que Boletus Bellini Inz. selon Reichert représente une bonne espèce au même titre que Ixocomus Boudieri Quél. Mais des recherches personnelles ,effectuées en Sicile établissent indubitablement qu'Inzenga créa son $B$. Bellini lors d'une communication à l'Académie de Catane le 3 Septembre 1876. 
Notre propos réel se limitait ici à Ixocomus Boudieri Quél. dont nous souhaiterions avoir donné une description complète et une meilleure connaissance, grảce au bolet de Mont-Pins avec lequel il s'identifie de façon définitive. Certes d'autres plantes ont aussi retenu notre attention, mais il s'agissait, par nécessité de le dégager de ses voisins les plus proches et les plus mal connus: Boletus leptopus Pers., B. littoralis Bouchet, B. Bellini Inz.

Toutefois cet exposé ne saurait encore s'achever sans qu'il soit rendu compte des affinites profondes de notre Ixocomus Boudieri avec le Boletus placidus, créé en 1861 par Bonorden, et affligé d'une bonne douzaine de noms différents. Kon rad (1927, p. 199), en parle dans son article. Konrad et Ma u bI anc (1927, PI. 415) synonymisent Boletus Bellini Inz. et Ixocomus Boudieri Quél. Ils rapportent lopinion de R. Maire selon lequel Bellini, confondu aussi avec Boudieri, et Boletus placiclus „sont deux races différentes d'une méme espèce adaptées à des hötes différents", à savoir le pin d'Alep et le pin Weymouth.

Quand on aura rappelé le chapeau blanchâtre des deux espèces (au moins au début chez Ixocomus Boudieri), leur granulation également intense, leurs réactions chimiques identiques *, comment ne pas expliquer par cette grande affinité, et du fait que placidus Bon. est une espèce montagnarde adaptée au froid, la remarquable vocation hivernale d'I. Boudieri Quél.

On nous permettra même de supposer, dans la mesure où Boletus placidus Bon., considéré comme espèce adventice, a suivi les pins Weymouth depuis 1705 dans leur transplantation d'Amérique en Europe, que 1. Boudieri Quél. pouvait, et peut se trouver aussi de l'autre cóté de l'Atlantique? Il nous serait agréable que des mycologues américains viennent confirmer notre hypothèse.

Si le rapprochement d'I. Boudieri Quél avec Boletus placidus Bon. s'impose de soi-méme, il est pourtant d'illustres spécialistes des bolets, tel S inger (1965, p. 78) qui, sans contester des affinités incontestables, affirme l'individualité des deux plantes en tant qu'espèces: „der echte Suillus boudieri ... weder mit $B$. granulatus noch mit $S$. placidus konspezifisch ist".

Prise de position sans équivoque qui nous permet de nous résumer ainsi:

- Kithner et Romagnesi (1953) signalent une réaction instantanée en rose rouge de la chair de Botutus placidus Bon. au contact de lammoniaque. Singer (1965, p. 75), constate comme nous, une réaction lilas: „NH4 $\mathrm{OH}$ bei Reife auf Hut lilaviole:t, auf Fleisch lila; auf Poren blau oder grau". 
D'une part entre Boletus Bellini Inz., espèce thermo-xérophile, nettement australe (Sicile et Palestine, se situent respectivement à la latitude de Bizerte et de Marrakech), strictement attachée aux Pinus halepensis; et d'autre part entre sa variété littoralis de type océanique, hygrophile, à pied plus développé, qu'on rencontre sur la façade atlantique de la France, uniquement sous Pinus maritima - Ixocomus Boudieri Quel. apparaît, de façon spécifique et géographique, dans une position centrale.

Moins exclusif sans doute dans ses rapports symbiotiques (nous l'avons cueilli dans des bois de Pinus halepensis mêlés de $P$. pinea, et Singer ainsi que Maire, l'auraient même récolté sous $P$, sylvestris, selon Reichert1), mais surtout fréquent sous $P$. halepensis, Ixocomus Boudieri Quél. se propose comme une bonne espèce, tout à fait légitime et stable. qu'on ne saurait plus contester désormais, ni confondre en particulier avec Boletus Bellini ou B. leptopus.

Nous formons le voeu que la diagnose ci-après, le fixe définitivement dans la nomenclature, et lui donne carrière dans les flores modernes.

\section{Suillus Boudieri (Quél.) Marchand nov. comb.}

Diagnosis: pileo $8-10 \mathrm{~cm}$, carnoso, convexo deinde convexoplano vel plus vel minus gibboso, primum albo deinde argilaceo surdo, postremo rufo-castaneo nonnunquam paene violaceo ad marginem, viscoso, margine involuta, acuta, excedenti, diutissime alba, postremo flava-citrina. Stipite $3 \mathrm{~cm}$ nonnunquam brevissimo $1,5 \mathrm{~cm}$ forma tronci-coni, in medio $2,2 \mathrm{~cm}$ sursum cum $1,2 \mathrm{~cm}$ paene deorsum, tecto in omni longitudine rubris carbunculosis granulis deinde nigrescentibus, in citrino recessu sursum, deorsum paene albo. Sine velo. Mycelio abundanti sive paene albo, sive rosa violaceo aut salmoneo. Carne alba nacrea, flava. citrina in superficie et in hymenio, non caerulescenti. Odore forti fructum redolenti mixto coco. Sapore grato fungitosa Marasmi oreadis. Tubis aperte decurrentibus initio, postremo adnato sinuoso, brevibus, inseparabilibus, flavis fere virentibus. Poris minutis, pallidis et surdis, postremo fere flavidis, fere olivaci, neque caeruleis dum premuntur, exsudantibus eosdem granulatus atque stipes. Sporis in cumulo flavidis argilaceis surdis. Sporis laevibus ellipticis-fusiformis, flavis maximeque pallentibus s. $m, 9,25 \times 3,75$.

Habitatio: Sub Pinis halepensibus, ab automno usque ver, aut gregarius aut singularis, ut dicitur Monte-Pinus, haud procul a Perpignane, in Gallia.

Ty pu s: Ixocomus Boudieri Quét. Bull. Soc. Mycol. Fr. t. 3, F. 3. 1878. 


\section{BIBLIOGRAPHIE}

Bouchet P. 1958, Boletus littoralis species nova du groupe Plorans. Bull. Trim.

Soc. Bot. Centre-Ouest, sér. 2, No 13, p. 32.

B resadola J., 1927-33. Iconographia Mycologica, Mediolani.

Gilbert E. J., 1931, Les Bolets, Paris.

Gilbert E. J, 1936, Notules sur les Bolets IV. Ixocomus leptopus Persoon.

Bull, Sac. Myc. Fr. 52:252-253.

In zenga G., 1869; 1879, Fungt Stciliant. Cent. I, 95 p. 8 Fl.; Cent. II. 79 p. II $\mathrm{Pl}$.

Inzenga G., 1878, Botetus Bellini Inz. Atti Acad. Gioen, 12:111-113, Catania. Kon rad P, 1927, Notes critiques sur quelques champignons du Jura. Bull. Soc. Myc. Fr. 43:199-204.

Konrad P. et Maublane A., 1924-1937, Icones selectae Fungorum, Paris. $\mathrm{K}$ üner R. et Romagnesi H., 1953, Flore analytique, Paris.

$\mathrm{P}$ a r dé L., Les Conifères, Paris.

Patouillard N., 1883-1889, Tabulae analyticae Fungorum.

Persoon C. H., 1822-1828, Mycologia europaea, Erlangen.

Qu el et L. 1878, Art. Boletus Botudieri Q. (Texte et Icône 1-ère), Bull. Soc. Myc.

Fr., 3.

Quélet L., 1888, Flore Mycologique, Paris,

Reichert I. 1940, Studies on Nushrooms and other Fungt of the Forests of

Palestine, Journ. of Bot. univ. Rehovot, 3:209-224; 233-258.

Rostkovius F. W. Th., 1838-1848, Pilze, in J. Sturm, Deutschland Flora. Nuremberg.

Saccardo P. A., 1915-1916, Hymeniales, in Flora ttalica cryptogama.

Sehaeffer J., 1952, Russula-Monographiae. Bad Heilbrunn Obb. (auf hymenialcystiden p. 35).

Singer R,, 1965, Die Röhrlinge. T. 1, 1967 T. II, Bad Heilbrunn Obb.

Mato znany maślak Suillus Boudieri (Quél.) Marchand

\section{Streszezenie}

Autor omawia szczególowo cechy Ixocomus Boudieri opisanego przez Quéleta, póżniej wielokrotnie nie rozróźnianego. Porównuje go $\mathrm{z}$ bliskimi gatunkami, Boleuts Bellini Inz., B. leptopus Pers. i B. littoratis Bouchet. Dochodzi do wniosku, że jest to gatunek dobry; robl nową kombinacje: Sullus Boudieri (Quél.) Marchand. 